Editor's Note: These short, critical reviews of recent papers in the Journal, written exclusively by graduate students or postdoctoral fellows, are intended to summarize the important findings of the paper and provide additional insight and commentary. For more information on the format and purpose of the Journal Club, please see http://www.jneurosci.org/misc/ifa_features.shtml.

\title{
EphrinA and TrkB Interact to Promote Axon Branching
}

\author{
B. Ian Hutchins ${ }^{\star}$ and $\mathrm{Li} \mathrm{Li}^{*}$ \\ Neuroscience Training Program, University of Wisconsin-Madison, Madison, Wisconsin 53706 \\ Review of Marler et al. (http://www.jneurosci.org/cgi/content/full/28/48/12700)
}

In the developing nervous system, projection neurons extend axons tipped with motile growth cones that are responsible for navigating to their appropriate targets while avoiding inappropriate targets. In chicks and mammals, axons use a complex strategy called delayed interstitial branching to accomplish this goal. Rather than growing directly into the appropriate termination zone, axons grow past their targets, and then selectively extend collateral branches onto and around their targets. Finally, exuberant branches and the primary axon are pruned, leaving the appropriate connections in the final termination zone.

How do axons restrict their branching to their appropriate termination zone? The retinotectal system has been studied extensively to address this question (for review, see McLaughlin and O'Leary, 2005). Retinal ganglion cell (RGC) axons enter the anterior tectum and project to the posterior tectum. Axons from temporal retina preferentially branch into the anterior tectum, while axons from nasal retina branch into the posterior tectum. It is thought that axons achieve this selective branching by responding to gradients of axon guidance molecules that suppress branching at inappropriate locations. Members of the ephrinA family of guid-

Received Jan. 15, 2009; revised Feb. 20, 2009; accepted Feb. 23, 2009.

This work was supported by National Research Service Award GM801642 to B.I.H. and a Herman I. Shapiro Fellowship to L.L.

*B.I.H. and L.L. contributed equally to this work.

Correspondence should be addressed to either B. Ian Hutchins or Li Li, Neuroscience Training Program, 1300 University Avenue, Madison, WI 53706,E-mail: bihutchins@wisc.edu or lli5@wisc.edu.

D0I:10.1523/JNEUROSCI.0238-09.2009

Copyright $\odot$ 2009 Society for Neuroscience $\quad$ 0270-6474/09/294329-03\$15.00/0 ance cues are key mediators of this process, and are expressed in a high-to-low gradient from posterior to anterior tectum. The receptor for ephrinA, named EphA, is expressed in a high-to-low gradient from temporal to nasal on retinal axons. Extension of collateral branches is thought to be locally suppressed along the axon once a threshold level of EphA activation is reached. Temporal axons, which express a higher concentration of EphA receptors, require less ephrinA to suppress branching than nasal axons, and therefore branch anterior to their nasal counterparts. While this model explains why temporal axons only branch in anterior tectum, it does not explain why nasal axons preferentially branch in posterior tectum-a single branch-suppressing gradient would lead nasal axons to branch along the entire anteroposterior extent of the tectum. Therefore a second gradient is necessary to explain the suppression of nasal axon branching in anterior tectum.

Interestingly, retinal axons express ephrinA5 in addition to EphA. In contrast to EphA, which is more highly expressed on temporal axons, the ephrinA gradient is highest on nasal axons and lowest on temporal axons. This countergradient of ephrinA suggested that ephrinA might act as a receptor on retinal axons rather than a ligand. Such a role has been demonstrated in the closely related ephrinB and EphB family proteins, which have both been shown to act as ligand and receptor (McLaughlin et al., 2003). For this to be the case, EphA should be expressed in a gradient in the tectum, and axons should respond to EphA as a guidance cue. In- deed, one member of the EphA family, EphA7, is expressed in a high-to-low anterior-to-posterior gradient in the superior colliculus (SC) (Rashid et al., 2005), the mammalian homolog of the tectum. EphA7 was not detected in retinal axons. Furthermore, retinal ganglion cell axons in culture avoid stripes of EphA7 laid down on the substrate, and nasal axons in EphA7-deficient mice aberrantly project to a second, anterior termination zone.

Although the expression patterns ephrinA and EphA7 are compatible with a model in which ephrinA acts as a receptor on retinal axons, unlike the transmembrane ephrinB proteins, ephrinAs are GPI linked, lacking an intracellular domain. This makes it unlikely that they act as a genuine receptor. How, then, do ephrinAs transduce extracellular EphA7 gradients into spatially instructive intracellular signals? Marler et al. (2008) address this question in a recent article in The Journal of Neuroscience.

Marler et al. (2008) hypothesized that ephrinA might act indirectly, as a coreceptor for a second signaling pathway. TrkB, a receptor for brain-derived neurotrophic factor (BDNF), was a good candidate since BDNF promotes branching of RGC axons. Both overexpression of ephrinA5 and treatment with BDNF increased the number of axon branches in retinal axons in vitro [Marler et al. (2008), their Fig. $1 F, G]$. Interestingly, when BDNF was applied to the neurons that overexpressed ephrinA5, axon branching was further increased [Marler et al. (2008), their Fig. $1 F, G]$. Although EphA7-Fc, which acti- 
vates ephrinA5, did not interfere with ephrinA5-enhanced axon branching, EphA7-Fc abolished the ability of BDNF to further enhance axon branching in the presence of ephrinA5 [Marler et al. (2008), their Fig. $1 G$ ]. This result raises the possibility that ephrinA5 might act through BDNF-dependent and -independent pathways, although further study would be required to confirm this possibility.

To test whether TrkB is involved in BDNF- or ephrinA5-promoted axon branching, Marler et al. (2008) knocked down TrkB using RNA interference. Knockdown of TrkB abolished BDNFenhanced retinal axon branching [Marler et al. (2008), their Fig. 2 B, C] and diminished increased axon branching evoked by ephrinA5 [Marler et al. (2008), their Fig. $2 C]$. These results demonstrate that ephrinA5 interacts with BDNF/TrkB signaling to promote axon branching.

Marler et al. (2008) next tested whether ephrinA5 directly interacts with Trk family proteins by using coimmunoprecipitation assays. EphrinA5 coimmunoprecipitated with the three members of the Trk family but not with PDGFR (another receptor tyrosine kinase), when overexpressed in $\mathrm{CHO}$ cells [Marler et al. (2008), their Fig. 4A, $B$ ]. This suggests that ephrinA5 and Trk receptors form a complex. Is this complex necessary for their functional interaction? By deleting different regions of the TrkB receptor, the authors identified that the CC2 domain of TrkB receptors is necessary for the interaction between ephrinA5 and TrkB [Marler et al. (2008), their Fig. 4D]. Furthermore, insertion of the CC2 domain into an unrelated receptor, Robo2, induced a novel interaction between Robo2 and ephrinA5 [Marler et al. (2008), their Fig. 4E]. Thus, the CC2 domain is also sufficient to induce the interaction between TrkB receptors and ephrinA5. When an isolated CC2 domain was overexpressed in RGCs, it abolished the interaction between ephrinA 5 and Trk and consequently diminished increased axon branching evoked by BDNF, ephrinA5, and both [Marler et al. (2008), their Fig. $4 F, G]$. These results demonstrated for the first time the interaction between ephrinA5 and Trk receptors and provided a model of how ephrinA5 acts as a receptor in the reverse signaling of EphA/ephrinA.

Previous studies have revealed roles for the Ras/MAP kinase, PI-3 kinase/Akt, and phospholipase C pathways in neurotrophin signaling (Segal, 2003). Which neurotrophin signaling pathway is initiated after ephrinAs interacts with Trk receptors? Since overexpression of Trk recep-
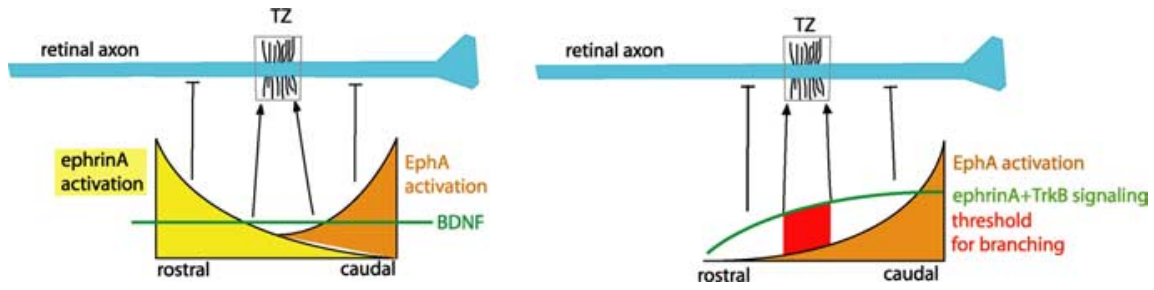

Figure 1. Left, Two gradients EphA and ephrinA in the tectum activate axonal ephrinA in the rostral tectum and axonal EphA in the caudal tectum, respectively, to suppress branching. BDNF promotes branching uniformly, and branching occurs where axonal ephrinA and EphA activation reaches a minimum. Adapted from Figure $8 B$ of Marler et al. (2008). Right, An alternative model in which only axonal EphA suppresses branching, while axonal ephrinA/TrkB promote branching. Tectal EphA (highest in rostral tectum) disrupts ephrinA/TrkB signaling, so ephrinA/TrkB activity increases from rostral to caudal. Branching occurs once an intracellular threshold is reached.

tors in CHO cells activated Trk receptors, the authors switched to another cell line, PC 12 cells, which has endogenous TrkA with low activity that is inducible by NGF. NGF treatment induced stronger downstream activation of Akt in the presence of ephrinA than in its absence, whereas the two other pathways were not affected [Marler et al. (2008), their Fig. 6A, B], suggesting that ephrinA5 binding to Trk specifically enhances Akt activation. Furthermore, neurotrophin induced interaction between ephrinA5 and TrkA [Marler et al. (2008), their Fig. 5A]. EphA7-Fc blocked ephrinA5-augmented Akt activation under stimulation by NGF [Marler et al. (2008), their Fig. 6C], which explains why application of EphA7-Fc diminished ephrinA5-augmented axon branching in the presence of BDNF [Marler et al. (2008), their Fig. 1G].

This study was the first to show that ephrinAs expressed on RGC axons interact with the BDNF/TrkB signaling pathway to promote axon branching, and tectal EphA7 antagonizes this process. Based on these results, Marler et al. (2008) draw two conclusions: first, in the absence of tectal EphA7, axonal ephrinA binds to TrkB and potentiates BDNF/TrkB signaling, and second, when activated by EphA7 in the anterior tectum, axonal ephrinA suppresses BDNF-induced axon branching [Marler et al. (2008), their Fig. 8]. The first conclusion is well supported by the experimental evidence presented in this paper. However, some key questions remain about the second conclusion. For example, is the EphA7/ephrinA interaction really inhibiting $\mathrm{BDNF}$-induced axon branching? An alternative explanation for the results of Marler et al. (2008) is that ephrinA only functions as a branchpromoting coreceptor for TrkB, and EphA7 merely interrupts this branchpromoting interaction. Both mechanisms would lead to lower levels of branching in the anterior tectum where EphA7 concen- trations are high, but each explanation makes different predictions about the downstream signaling mechanisms. In our alternative model, BDNF/TrkB signaling alone is insufficient to cause axon branching in the tectum, but axonal ephrinA enhances TrkB signaling such that an intracellular signaling threshold is overcome and branching occurs (Fig. 1, right). In contrast, the model proposed by Marler et al. (2008) proposes that BDNF/ TrkB signaling is sufficient to induce branching in the tectum, but that EphA7/ ephrinA somehow suppresses this baseline level of axon branching (Fig. 1, left). Indeed, one key result from Marler et al. (2008) is consistent with our alternative hypothesis: if EphA7 activates ephrinA to suppress branching, then axons overexpressing ephrinA and treated with EphA7 should have significantly fewer branches than those not treated with EphA7, but they do not [Marler et al. (2008), their Fig. $1 G]$. This result is inconsistent with the branch-suppression hypothesis favored by Marler et al. (2008), and highlights the need for further study.

Since both models predict the same retinotectal branching pattern (Fig. 1), how can we differentiate between these two models? Two recent studies may provide mechanistic insights to the signaling pathways investigated by Marler et al. (2008). A study by Lim et al. (2008) demonstrated that ephrinA on RGC axons also interacts with $\mathrm{p} 75$, another BDNF receptor. EphA7/ephrinA complex activated p75 to mediate repulsion in vitro, and p75-null axons failed to avoid substratebound EphA7. This result could be interpreted as supporting the hypothesis that EphA7/ephrinA actively suppresses branching. However, RGC axons from p75-deficient mice did not replicate the EphA7-null phenotype: rather than projecting to both anterior and posterior termination zones as in the EphA7-deficient mice (Rashid et al., 2005), nasal RGC ax- 
ons shifted or expanded to slightly more anterior positions (Lim et al., 2008). If EphA7 acts via p75 to suppress branching, then similar guidance errors should be observed in both knock-outs.

If the results from $\mathrm{p} 75$-deficient mice do not support the hypothesis that EphA7/ephrinA actively suppresses branching, how do they fit with our alternative model? A recent study by Singh et al. (2008) showed that p75 inhibits the Trk signaling pathway, including the PI3 kinase pathway in peripheral axons. It is possible that p75 activation does not reduce branching per se, but rather inhibits $\mathrm{BDNF} /$ TrkB signaling. In this case, removing EphA7 from the tectum would disinhibit the branch-promoting ephrinA/TrkB interaction and would also relieve the downstream inhibition of PI3 kinase from EphA7/ephrinA/p75 signaling. This would lead to dramatic guidance errors to anterior tectum/SC, as seen in the EphA7 knock-out mouse (Rashid et al., 2005). In contrast, removing p75 from RGC axons would relieve inhibition of signaling downstream of BDNF/TrkB but would not disinhibit the ephrinA/TrkB interaction. This might be expected to lead to less severe guidance errors. One way to test between the two models discussed here (Fig. 1) would be to overexpress p75 in nasal RGCs and treat them with EphA7 in the absence of BDNF. If EphA7/ephrinA/p75 actively suppresses branching, there should be fewer branches per axon for cells overexpressing p75 compared with controls, in the presence of EphA7. On the other hand, if EphA7/ephrinA/p75 merely inhibits BDNF/TrkB signaling once activated, then there should be no difference between these two groups.

Although much still needs to be done to fully understand the mechanisms of retinotopic mapping, the results by Marler et al. (2008) offer important insights into complex interactions between different guidance cues and bidirectional signaling of EphAs/ephrinAs by providing the first evidence that EphA/ephrinA interacts with $\mathrm{BDNF} / \mathrm{TrkB}$ signaling to regulate axon branching in the retinotectal system.

\section{References}

Lim YS, McLaughlin T, Sung TC, Santiago A, Lee KF, O'Leary DD (2008) p75(NTR) mediates ephrin-A reverse signaling required for axon repulsion and mapping. Neuron 59:746-758.

Marler KJ, Becker-Barroso E, Martínez A, Llovera M, Wentzel C, Poopalasundaram S, Hindges R, Soriano E, Comella J, Drescher U (2008) A TrkB/EphrinA interaction controls retinal axon branching and synaptogenesis. J Neurosci 28:12700-12712.

McLaughlin T, O'Leary DD (2005) Molecular gradients and development of retinotopic maps. Annu Rev Neurosci 28:327-355.

McLaughlin T, Hindges R, Yates PA, O'Leary DD (2003) Bifunctional action of ephrin-B1 as a repellent and attractant to control bidirectional branch extension in dorsal-ventral retinotopic mapping. Development 130:2407-2418.

Rashid T, Upton AL, Blentic A, Ciossek T, Knöll B, Thompson ID, Drescher U (2005) Opposing gradients of ephrin-As and EphA7 in the superior colliculus are essential for topographic mapping in the mammalian visual system. Neuron 47:57-69.

Segal RA (2003) Selectivity in neurotrophin signaling: theme and variations. Annu Rev Neurosci 26:299-330.

Singh KK, Park KJ, Hong EJ, Kramer BM, Greenberg ME, Kaplan DR, Miller FD (2008) Developmental axon pruning mediated by BDNF-p75NTR-dependent axon degeneration. Nat Neurosci 11:649-658. 